\title{
Estudo Comparativo entre Misoprostol e Placebo para 0 Amadurecimento Cervical e Indução do Parto
}

\author{
A Randomized Trial of Misoprostol and Placebo for \\ Cervical Ripening and Induction of Labor
}

Renata Murad Macedo, Ivete de Ávila, Manuel Maurício Gonçalves

\begin{abstract}
RESUM0
Objetivo: avaliar a eficácia e segurança da aplicação intravaginal de misoprostol para amadurecimento cervical e indução do parto em gravidez a termo quando comparado com placebo.

Pacientes e Métodos: cinqüenta e uma mulheres com gestação de alto risco, a termo, $e$ cérvix imatura foram alocadas em estudo duplo cego para aplicação de misoprostol intravaginal (40 $\mathrm{mg}$ de $4 / 4 \mathrm{~h}$ ) ou placebo intravaginal $(4 / 4 \mathrm{~h}$ ).

Resultados: entre as 51 pacientes estudadas, 32 receberam misoprostol e 19 receberam placebo. Os grupos foram homogêneos quanto à idade materna, idade gestacional, paridade $e$ indicação para indução ( $p>0,05$ ). No grupo Misoprostol observamos $87,5 \%$ de eficácia $e$ no grupo placebo $21,1 \%$ de eficácia ( $p=0,0000087$ ). Em relação à via de parto, no grupo Misoprostol 75\% dos partos foram vaginais e 25\% cesáreos, Já no grupo placebo, apenas $32 \%$ foram partos vaginais e 68\% cesáreos ( $p=0,0059)$. O Apgar neonatal foi semelhante em ambos grupos.

Conclusão: misoprostol se apresentou extremamente eficaz e seguro no amadurecimento cervical e indução do parto, surgindo como nova opção em obstetrícia em gestações de alto risco, a termo, com cérvix imatura e com necessidade de resolução do parto a curto prazo.
\end{abstract}

PALAVRAS-CHAVE: Trabalho de parto. Parto Induzido. Prostaglandina. Resolução da gravidez. Gestação de alto risco.

\section{Introdução}

A cérvix uterina altera-se consideravelmente no final da gestação, tornando-se nas últimas semanas mais amolecida, edemaciada, elástica e consequentemente mais distensivel. Este processo natural é denominado amadurecimento cervical, sendo parte integrante e fundamental das

Hospital dos Servidores do Estado de Minas Gerais - IPSEMG Correspondência:

Renata Murad Macedo

Rua Levindo Lopes, 231 Apto 205. Bairro Savassi.

30140-170 - Belo Horizonte - MG alterações que precedem o trabalho de parto. A indução do parto, seja mecânica ou farmacológica, torna-se freqüentemente prolongada e complicada quando a cérvix é considerada não-favorável ou imatura ${ }^{7}$.

Ao final da gestação a estrutura cervical é alterada a partir de dois eventos paralelos. Inicialmente ocorre aumento da concentração de ácido hialurônico e água. Concomitantemente há diminuição do dermatan sulfato levando à ruptura das ligações entre fibrilas colágenas, ruptura do colágeno e redirecionamento das fibras. Em resumo ocorre uma reorganização da matriz extracelular 
tornando a cérvix amolecida e distensível ${ }^{7}$.

A indução do parto realizada quando a cérvix não está madura é associada a uma alta incidência de trabalho de parto prolongado, estresse fetal, parto instrumental e cesariana. Vários agentes têm sido usados para amadurecer a cérvix uterina e induzir o parto, entre eles as prostaglandinas e seus análogos, que têm apresentado ótimos resultados ${ }^{5,9,10}$.

Margulies et al. foram os primeiros a utilizar o misoprostol - análogo da prostaglandina $\mathrm{E}_{1}$ - para indução do parto, na tentativa de encontrar uma droga eficaz, segura e de baixo custo, quando comparada à prostaglandina $\mathrm{E}_{2}\left(\mathrm{PGE}_{2}\right)^{8}$.

Clínica e experimentalmente tem-se demonstrado a eficácia da $\mathrm{PGF}_{2 \alpha}, \mathrm{PGE}_{2}$ sintética e PGE 1 sintética no amadurecimento cervical e indução do trabalho de parto, provando serem estas substâncias agentes farmacológicos promissores em obstetrícia. No presente estudo efetivamos uma observação clínica comparativa, randomizada, duplo cego, para avaliar a eficácia e segurança do análogo prostaglandina $\mathrm{E}_{1}$ - misoprostol no amadurecimento cervical e indução do parto em gestações de alto risco, a termo.

\section{Pacientes e Métodos}

Utilizamos óvulos à base de glicerina, contendo ou não misoprostol ( $40 \mu \mathrm{g}$ ), de acordo com o grupo placebo ou grupo da droga. Os óvulos foram obtidos a partir da manipulação farmacêutica de comprimidos de Cytotec $\AA$ (Biolab - Searle Lab. Incorp.), comercialmente distribuídos com dose de $200 \mu \mathrm{g}$. A dosagem foi padronizada de maneira empírica, baseada na experiência prévia dos pesquisadores.

O critério básico para avaliação da eficácia do óvulo foi eminentemente clínico. Utilizamos o indice proposto por Bishop ${ }^{2}$, usado em quase todos os relatos literatura mundial sobre prostaglandinas na indução do parto. Este índice avalia as condições próprias da cérvix (posição, consistência, apagamento, dilatação) associado à altura da apresentação do polo cefálico fetal, às quais são dadas notas de zero a três que somadas indicam a indutibilidade ou não da cérvix. O índice ideal para indução do parto é maior ou igual a 9, sendo aceitável um índice maior ou igual a 6 .

Foram incluídos no estudo gestantes com indicações clínicas ou obstétricas para indução do parto. Consideramos como indicação as seguintes situações:

a) Doença hipertensiva específica da gravidez

\section{(DHEG)}

b) Hipertensão arterial crônica

c) Diabetes mellitus

d) Diabetes gestacional

e) Retardo de crescimento intra-uterino

f) Gestação prolongada (pós-datismo)

g) Isoimunização materna pelo fator Rh.

Também determinamos critérios de inclusão e exclusão conforme características próprias maternas ou da gestação.

Foram considerados critérios de inclusão:

a) Indicação clínica ou obstétrica para indução

b) Gravidez única com idade gestacional de 37 semanas ou mais, sendo calculada pela data da última menstruação confiável ou exame ultrassonográfico de $1^{\circ}$ ou $2^{\circ}$ trimestre

c) Apresentação fetal cefálica

d) Membranas integras

e) Índice Bishop menor ou igual a 6

f) Cardiotocografia basal reativa e/ou perfil biofísico fetal maior ou igual a 8

g) Consentimento da paciente.

Foram considerados critérios de exclusão:

a) História prévia de cesariana ou cirurgia uterina; b) Presença de sofrimento fetal crônico grave oligoidrâmnio acentuado (bolsão de líquido amniótico menor que $2 \mathrm{~cm}$ )

c) Presença de hemorragia genital

d) Presença de infecção genital ativa

e) História clínica de asma, cardiopatia ou glaucoma f) Presença de febre

g) História de alergia ao misoprostol ou outras prostaglandinas

h) Uso recente de antiinflamatórios não-esteróides (até 6 horas antes).

As gestantes selecionadas foram então protocoladas conforme a padronização prévia. Após a coleta de dados era feita a avaliação do bemestar fetal por meio de cardiotocografia basal e/ou ultra-sonografia, seguida de avaliação dos dados vitais maternos (pulso, pressão arterial, temperatura e freqüência respiratória).

As pacientes selecionadas eram então alocadas ou no grupo misoprostol, ou no grupo placebo, por meio de sorteio aleatório. Por ser estudo duplo cego, não se conhecia em qual grupo se utilizava a medicação ou placebo.

Um total de 51 pacientes foram distribuídas em dois grupos e as condições da cérvix uterina eram avaliadas através do índice de Bishop.

A primeira aplicação era feita no ambulatório, com uso de óvulo intravaginal, de acordo com o grupo sorteado. A paciente inicialmente inserida em um grupo, permaneceria nele até se completar 
todas as doses.

No grupo misoprostol, com 32 pacientes, estas recebiam um óvulo de $40 \mu \mathrm{g}$ para amadurecimento cervical. Após 24 horas, a paciente retornava e recebia óvulos de $40 \mu \mathrm{g}$ a cada 4 horas (máximo de 5 doses), perfazendo um total de 6 doses.

No grupo placebo, com 19 pacientes, estas recebiam um óvulo de glicerina para amadurecimento cervical. Após 24 horas, a paciente retornava e recebia óvulos de glicerina a cada 4 horas (máximo de 5 doses), perfazendo um total de 6 doses.

Após a $1^{\mathrm{a}}$ aplicação a paciente era mantida em repouso, em decúbito lateral esquerdo por 1 hora, e então liberada para o domicílio após reavaliação dos dados vitais e do bem-estar fetal por meio da ausculta dos batimentos cardiofetais por sonar doppler.

Todas as pacientes eram reexaminadas após período de 24 horas, e se realizava uma nova avaliação da cérvix. A paciente então, era internada e submetida à indução do trabalho de parto com óvulos 4/4 horas. As pacientes seguiam o grupo em que foram protocoladas inicialmente.

A aplicação de óvulos era suspensa logo que a paciente iniciasse o trabalho de parto, com um mínimo de 3 contrações de 25 segundos em 10 minutos, ou se ocorresse amniorrexe espontânea das membranas, ou a qualquer sinal de prejuízo ao bem-estar materno e fetal.

\section{Resultados}

No período compreendido entre maio 96 a julho 97, estudamos 51 gestantes a termo com indicações clínicas e/ou obstétricas para indução do parto, e que preenchiam os critérios de inclusão e exclusão previamente estabelecidos. As indicações para indução estão resumidas no Tabela 1 .

Tabela 1 - Indicações para Indução do parto

\begin{tabular}{lcc}
\hline & $\begin{array}{c}\text { Grupo Caso } \\
\mathrm{n}=32\end{array}$ & $\begin{array}{c}\text { Grupo Controle } \\
\mathrm{n}=19\end{array}$ \\
\hline Pós-datismo & 24 & 15 \\
Diabetes & 0 & 1 \\
Gestacional & & \\
DHEG & 2 & 1 \\
HAC & 3 & 1 \\
Isoimunizaçao Rh & 0 & 1 \\
Eletiva & 2 & 0 \\
CIUR & 1 & $\mathbf{1 9}$ \\
Total & $\mathbf{3 2}$ & 0
\end{tabular}

É importante ressaltar que as duas pacientes com indicação eletiva foram alocadas em razão da esquizofrenia, internadas neste hospital, sendo indicada a indução por tentativa de suicídio, em um caso. No outro, havia feto anencéfalo com gestação a termo. Os dados a respeito das pacientes estão representados nas Tabela 2. Estes demonstram que os grupos são homogêneos quanto à idade materna, idade gestacional e paridade.

Tabela 2 - Idade materna, Idade gestacional e número de primíparas nos dois grupos. Intervalo, média e desvio padrão.

\begin{tabular}{|c|c|c|c|c|c|c|}
\hline & Total & \multicolumn{2}{|c|}{ Caso $n=32$} & \multicolumn{3}{|c|}{ Controle $n=19$} \\
\hline & $\mathrm{p}$ & Intervalo & Média & Interv & & Média \\
\hline Idade Materna & 0,57 & $15-44$ & $27,2 \pm(1,31)$ & $16-38$ & 27 & $6 \pm(1,5)$ \\
\hline \multicolumn{7}{|l|}{ Idade } \\
\hline \multirow[t]{2}{*}{ gestacional } & 0,98 & $37-42$ & $40,4 \pm(0,23)$ & $37-42$ & & $4 \pm(0,30)$ \\
\hline & $\mathrm{p}$ & $\mathrm{n}$ & $\%$ & $\mathrm{n}$ & & $\%$ \\
\hline Primípara & 0,46 & 19 & 59,4 & 14 & & 73,7 \\
\hline
\end{tabular}

Os resultados eram considerados positivos, se a gestante apresentasse índice Bishop maior que 6 e/ou se iniciasse o trabalho de parto após o uso dos óvulos.

A Tabela 3 ilustra os resultados e mostra eficácia em $87,5 \%$ no grupo misoprostol e em $21,1 \%$ no grupo placebo. Observa-se na que das 28 pacientes que apresentaram resultados positivos no grupo misoprostol, 27 iniciaram o trabalho de parto e 22 apresentaram índice Bishop maior que 6 com o uso dos óvulos.

Tabela 3 - Porcentagem de resultados positivos* em caca grupo.

\begin{tabular}{lcccccc}
\hline & Misoprostol $\mathrm{n}=32$ & & \multicolumn{2}{c}{ Placebo $\mathrm{n}=19$} & & Total $\mathrm{n}=51$ \\
\hline & $\mathrm{n}$ & $\%$ & $\mathrm{n}$ & $\%$ & $\mathrm{p}$ & OR (IC 95\%) \\
$\begin{array}{l}\text { Resultados } \\
\text { positivos }\end{array}$ & 28 & 87,5 & 4 & 21,1 & 0,0000087 & $26(4,6 \mathrm{a} 175)$ \\
\hline
\end{tabular}

*Resultados positivos: BISHOP $>6$ e/ou trabalho de parto

No grupo placebo, das 4 pacientes que apresentaram resultados positivos, 4 iniciaram o trabalho de parto e 3 apresentaram índice Bishop maior que 6 com o uso dos óvulos de glicerina.

As diferenças são estatisticamente significativas (Tabela 3), já que o valor de p é 
0,0000087, sendo o "odds ratio" de 26,25 e o intervalo de confiança entre 4,69 e 175,11. Observamos que a droga apresenta 26 vezes mais chances em amadurecer o colo e induzir o parto que o placebo. Observamos também um intervalo de confiança alargado em razão da amostra ser pequena, porém maior que 1 , o que lhe concede valor estatístico.

Em relação à via de parto, no grupo observamos $75 \%$ de parto vaginal e $25 \%$ de parto cesáreo e no grupo placebo a relação foi praticamente inversa: $31,6 \%$ de parto vaginal e $68,4 \%$ de parto cesáreo $\mathrm{p}=0,0059$ (Figuras 1 e 2). Com isso, observa-se que a medicação foi extremamente eficiente na diminuição do índice de cesarianas, naquelas pacientes que necessitam de interrupção da gravidez à curto prazo, e que apresentam cérvix imatura em situações em que a permanência fetal intra-útero pode acarretar prejuízo materno e fetal.

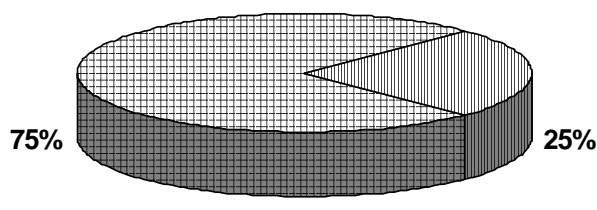

四 Cesárea $\mathrm{n}=8$ 囲 Vaginal $\mathrm{n}=24$

Figura 1 - Via de parto no grupo misoprostol em porcentagem

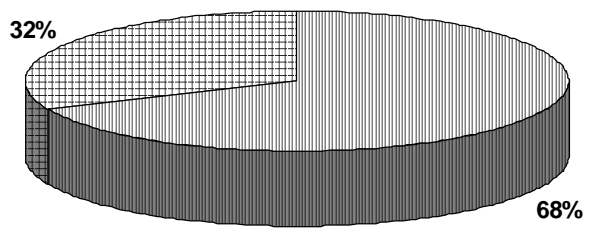

(n) Cesárea $\mathrm{n}=13$

$\boxplus$ Vaginal $n=6$

Figura 2 - Via de parto no grupo controle em porcentagem

As indicações para cesárea estão expostas na Figura 3 e evidenciam que a maior indicação para cesárea no Grupo Placebo foi cérvix em condições desfavoráveis para uso de ocitócico. No grupo misoprostol a indicação mais freqüente foi de falha na indução com misoprostol e ocitócico ocorrido em apenas 4 casos.

Os efeitos colaterais encontrados foram mínimos; sendo que no grupo misoprostol observamos: um caso de diarréia e vômitos $(3,1 \%)$ e dois casos de taquissistolia (6,3\%). O índice de Apgar dos recém-nascidos foi de 9 no $1^{\circ}$ minuto e 10 no $5^{\circ}$ minuto em ambos os casos. No grupo placebo ocorreu apenas um caso de taquissistolia (5,3\%), e o índice de Apgar do RN foi de 9 no $1^{\circ}$ minuto e 10 no $5^{\circ}$ minuto.

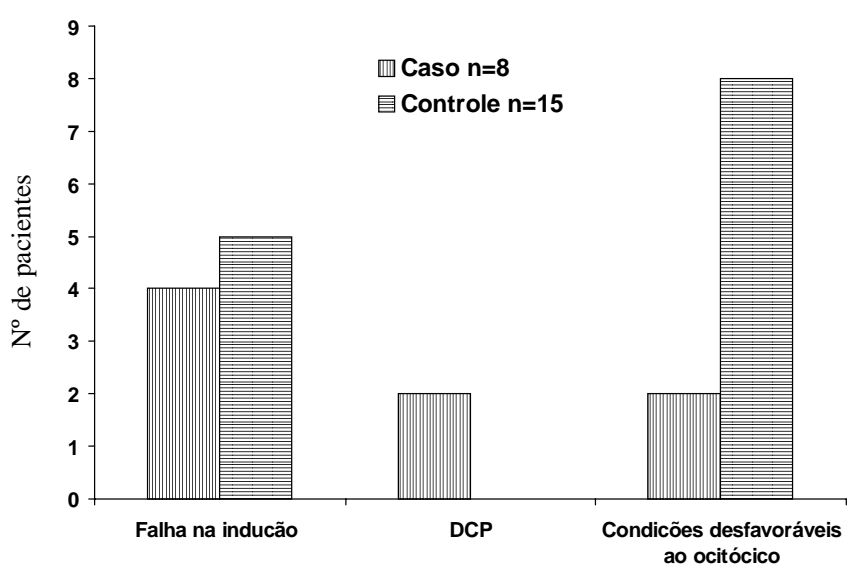

DCP: desproporção cefalopélvica

Figura 3 - Indicações de cesárea

Em relação ao Apgar dos recém-nascidos (Tabela 4), no grupo misoprostol 90,6\% dos RNs obtiveram Apgar no $1^{\circ}$ minuto maior que 7 e 93,8\% Apgar no $5^{\circ}$ minuto maior que 7 . Já no grupo placebo $94,7 \%$ dos RNs obtiveram Apgar no $1^{\circ}$ minuto maior que 7 e $100 \%$ Apgar no $5^{\circ}$ minuto maior que $7\left(\mathrm{p}=0,88\right.$ no $1^{\circ}$ minuto e 0,99 no $5^{\circ}$ minuto).

Tabela 4 - Indicação de Apgar no $1^{\circ}$ e $5^{\circ}$ minuto no grupo misoprostol e controle

\begin{tabular}{|c|c|c|c|c|c|}
\hline & Total & \multicolumn{2}{|c|}{ Caso $n=32$} & \multicolumn{2}{|c|}{ Controle $n=19$} \\
\hline & $\mathrm{p}$ & $\mathrm{n}$ & $\%$ & $\mathrm{n}$ & $\%$ \\
\hline Apgar 1'>7 & 0,88 & 29 & 90,6 & 18 & 94,7 \\
\hline Apgar 5'>7 & 0,99 & 30 & 93,8 & 19 & 100 \\
\hline
\end{tabular}

\section{Discussão}

Margulies et al. realizaram na Argentina a primeira experiência clínica com misoprostol aplicado a gestações a termo com fetos vivos. Utilizaram o misoprostol sob forma de comprimidos 
por via intravaginal, administrando doses de $50 \mu \mathrm{g}$ a intervalos de 2 horas, com máximo de $600 \mu \mathrm{g}$, a 56 gestantes com indicações clínicas ou obstétricas para indução do parto (gestação prolongada, hipertensão materna, isoimunização materno-fetal pelo fator $\mathrm{Rh}$ ). Obtiveram $73 \%$ de eficácia, com mínimos efeitos colaterais e $3,6 \%$ de hiperestimulação uterina, sem repercussão do ponto de vista neonatal ${ }^{8}$.

Kramer et al. realizaram um estudo comparativo randomizado entre misoprostol via intravaginal e ocitócico intravenoso, concluindo que o fármaco é um agente eficaz na indução do parto, com resultados e segurança de uso similares a ocitocina ${ }^{6}$.

Fletcher et al., na Jamaica, conduziram o primeiro estudo com misoprostol na forma de gel para aplicação intravaginal. Neste trabalho 24 gestantes receberam $100 \mu \mathrm{g}$ do gel de misoprostol comparando-se os resultados com os de 21 pacientes que receberam creme de etinilestradiol (placebo). Demonstraram acentuada diminuição da ocorrência de cesarianas e mínimos efeitos colaterais materno-fetais ${ }^{3}$.

Os mesmos autores conduziram a seguir um estudo comparativo entre o gel de misoprostol $(100 \mu \mathrm{g})$ e a $\mathrm{PGE}_{2}$ sintética - Dinoprostone ( $\left.3 \mu \mathrm{g}\right)$. O índice de parto vaginal foi similar nos dois grupos $(84,4 \%$ para o misoprostol e 90,3\% para o Dinoprostone). Os autores concluem que o análogo da $\mathrm{PGE}_{1}$ é tão eficaz quanto a $\mathrm{PGE}_{2}$ no amadurecimento cervical e indução do trabalho de parto a termo ${ }^{4}$.

Mais recentemente Chuck e Huffaker compararam a eficácia e segurança do misoprostol intravaginal e Dinoprostone intracervical concluindo que o primeiro é eficaz, comparável em segurança e com custo mais baixo ${ }^{1}$.

Ngai et al. investigam a eficácia de $200 \mu \mathrm{g}$ de misoprostol oral no amadurecimento cevical em pacientes com ruptura prematura de membranas com gestação à termo. Os resultados demonstram que a droga é efetiva e reduz a necessidade da infusão de ocitocina durante o trabalho do parto, além de diminuir a fase de latência. Não houve aumento dos efeitos colaterais, maternos ou morbidade perinatal ${ }^{11}$.

Nossas observações acerca de baixas dosagens de misoprostol vão de encontro a literatura, com eficácia semelhante nas taxas de indução de parto, demonstrando uma alta resolutividade do parto por via vaginal, e uma baixa incidência de efeitos colaterais materno-fetais.

Sendo assim, o misoprostol surge como nova opção em obstetrícia para amadurecimento cervical e indução do parto, principalmente em gestações a termo de alto risco, cérvix imatura e necessidade de resolução do parto a curto prazo.

\section{SUMMARY}

Objective: to determine the efficacy and safety of misoprostol for cervical ripening and induction of labor in pregnant women at term when compared with placebo.

Patients and Methods: fifty-one high-risk pregnant women at term, with unripe cervix, were allocated in a double-blind trial for treatment with intravaginal misoprostol $(40 \mu \mathrm{g}, 4 / 4 \mathrm{~h})$ or intravaginal placebo.

Results: thirty-two patients received misoprostol and 19 received placebo. The groups were homogeneous concerning maternal age, gestacional age, parity, and indication for induction $(p>0.05)$. In the misoprostol group the efficacy was $87.5 \%$ and in the placebo group $21.1 \%$ ( $p=0.0000087$ ). Regarding delivery, in the misoprostol group $75 \%$ had vaginal delivery and 25\% abdominal delivery, and in the placebo group only $32 \%$ had vaginal delivery and $68 \%$ abdominal delivery $(p=0.0059)$.The Apgar score was similar.

Conclusion: in this study misoprostol was effective and safe for cervical ripening and induction of labor.

KEY WORDS: Cervical ripening. Induction of labor. Prostaglandin. Pregnancy interruption. High-risk pregnancy.

\section{Referências}

1. Chuck FJ, Huffaker JB. Labor induction with intravaginal misoprostol versus intracervical prostaglandin $\mathrm{E}_{2}$ gel (Prepidil gel): Randomized comparision. Am J Obstet Gynecol 1995, 173:1137-42.

2. Bishop EH. Pelvic scpring for eletive induction. Obstet Gynecol 1964; 24: 266-8.

3. Fletcher HM, Mitchell S, Simeon D, Frederick J, Brown D. Intravaginal misoprostol as a cervical ripening agent. Br J Obstet Gynecol 1993; 100:641-3.

4. Fletcher H, Mitchell S, Frederick J, Simeon D, Brown D. Intravaginal Misoprotol versus Dinoprostone as cervical ripening and labor inducing agents. Obstet Gynecol 1994; 83:244-7.

5. Keirse MJN. Prostaglandins in preinduction cervical ripening. Metanalysis of wordwide clinical experience. J Reprod Med 1993; 38:89-100.

6. Kramer RL, Gilson GJ, Morrison DS, Martim D, Gonzalez JL, Qualls CR. A randomized trial of misoprostol and oxytocin for induction of labor: Safety and efficacy. Obstet Gynecol 1997; 89:387-91.

7. Leppert PC. Anatomy and physiology of cervical ripening. Clin Obstet Gynecol 1995; 38: 267-79.

8. Margulies M, Voto LS, Catuzzi P, Imaz FU. Inducción del trabajo de parto con un análogo de la $\mathrm{PGE}_{1}$. Prensa Med Arg 1991; 78:9-13. 
9.Massyl HY, Baker A, Shaugh OJM. A comparison of oral PGE2 tablets with intravenous oxytocin for stimulation of labor after premature rupture of membranes at term. Acta Obstet Gynecol Scand 1988; 67:703-9
10.Mundle WR, Young DC. Vaginal misoprostol for induction of labor: a randomized controlled trial. Obstet Gynecol 1996; 88:521-5.

11.Ngai SW, To WK, Lao T, Ho PC. Cervical priming with oral misoprostol in pre-labor rupture of membranes at term. Obstet Gynecol 1996; 87:923-6. 